\title{
EVALUACIÓN DE LA ESTABILIDAD DE CAROTENOIDES Y ACTIVIDAD ANTIOXIDANTE DE LA FLOR DE MASTUERZO (Tropaeolum majus L.) EN LA MICROENCAPSULACIÓN POR SPRAY-DRYING
}

\author{
Luis Juscamaita Fabián ${ }^{a}$, Tiffany Pérez Salvatierra ${ }^{a}$, Clara Espinoza Silva ${ }^{a}$, \\ Miguel Quispe Solano*b, Greta Hinostroza Quiñóneza, Omar Flores Ramos ${ }^{\mathrm{c}}$, \\ Galia Manyari Cervantes ${ }^{\mathrm{d}}$
}

\begin{abstract}
RESUMEN
Se realizó la extracción de carotenoides de los pétalos de flores amarillas de mastuerzo (Tropaeolum majus L.) con hexano, cloroformo y éter de petróleo a 12, 24 y 48 horas; el mayor rendimiento se obtuvo con cloroformo a 24 horas, el que se purificó para ser solubilizado con aceite de oliva. Luego se microencapsuló con maltodextrina y goma arábiga. La dispersión se llevó a un Mini Spray Dried B-290 a temperaturas de secado de $130{ }^{\circ} \mathrm{C}, 150{ }^{\circ} \mathrm{C}$ y $170{ }^{\circ} \mathrm{C}$; en el microencapsulado se evaluó el \% de recuperación de carotenoides totales encontrándose diferencia $(p<0,05)$ por efecto de la interacción del agente encapsulante y la temperatura de secado, los mayores \% de recuperación fueron $74,10 \%$ y $83,76 \%$ a 130 ${ }^{\circ} \mathrm{C}$ y $150{ }^{\circ} \mathrm{C}$, respectivamente, con goma arábiga; no se evidencia diferencia $(p>0,05)$ en la actividad antioxidante las que van de 393,00 a 470,97 $\mu$ mol-TE/g. de muestra. Con respecto al $\%$ de humedad, $\%$ de higroscopicidad, densidad aparente $\left(\mathrm{g} / \mathrm{cm}^{3}\right)$ y $\%$ de rendimiento se evidencia diferencia $(p<0,05)$. En el $\%$ de solubilidad no se evidencia diferencia $(p>0,05)$, la microcápsula de carotenoides puede ser usada como alimento funcional.
\end{abstract}

Palabras clave: Tropaeolum majus L., microencapsulación, carotenoides, actividad antioxidante, spray drying.

\footnotetext{
${ }^{\text {a }}$ Facultad de Ingeniería en Industrias Alimentarias, Universidad Nacional del Centro del Perú.

${ }^{\mathrm{b}}$ Escuela Profesional de Ingeniería Agroindustrial, Facultad de Ciencias Aplicadas, Universidad Nacional del Centro del Perú, quispe_miguelangel@hotmail.com

c Facultad de Ingeniería Mecánica, Universidad Nacional del Centro del Perú.

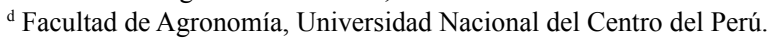




\title{
EVALUATION OF STABILITY OF CAROTENOIDS AND ANTIOXIDANT ACTIVITY OF NASTURTIUM FLOWERS (Tropaeolum majus L.) IN SPRAY-DRYING MICROENCAPSULATION
}

\begin{abstract}
Carotenoids were extracted from the petals of yellow nasturtium flowers (Tropaeolum majus L.) with hexane, chloroform and petroleum ether at 12, 24 and 48 hours; The highest yield was obtained with chloroform at 24 hours, which was purified to be solubilized with olive oil. It was then microencapsulated with maltodextrin and gum arabic. The dispersion was carried to a Mini Spray Dried B-290 at drying temperatures of $130{ }^{\circ} \mathrm{C}, 150^{\circ} \mathrm{C}$ and $170{ }^{\circ} \mathrm{C}$; In the microencapsulation the $\%$ recovery of total carotenoids was evaluated $(p<0.05)$ due to the interaction of the encapsulating agent and the drying temperature, the highest recovery rates were $74.10 \%$ and $83.76 \%$ At $130{ }^{\circ} \mathrm{C}$ and $150{ }^{\circ} \mathrm{C}$ respectively, with gum arabic; No difference $(p>0.05)$ was found in antioxidant activity ranging from 393.00 to $470.97 \mu \mathrm{mol}$ $\mathrm{TE} / \mathrm{g}$. shows. With regard to $\%$ humidity, $\%$ hygroscopicity, bulk density $\left(\mathrm{g} / \mathrm{cm}^{3}\right)$ and $\%$ yield difference was evident $(p<0.05)$. In the $\%$ solubility no difference $(p>0.05)$ is evident, the microcapsule of carotenoids can be used as functional food
\end{abstract}

Key words: Tropaeolum majus L., microencapsulation, carotenoids, antioxidant activity, spray drying.

\section{INTRODUCCIÓN}

Las exigencias de la alimentación, en la actualidad, se han incrementado respecto a la calidad (organoléptica, microbiológica y nutricional), así la demanda de productos de carácter nutracéutico y funcional va en aumento; debido a que mejoran la salud y/o previenen ciertas enfermedades.

Los colorantes generalmente empleados en la industria de alimentos son de carácter sintético, estos cumplen bien sus funciones de tinción, sin embargo no aportan valor nutritivo considerable. Recientemente, se ha puesto de manifiesto que la relevancia de los carotenoides va más allá, de que algunos de ellos poseían actividad provitamínica $\mathrm{A}$, al haberse demostrado que juegan un papel importante en la prevención de diversas enfermedades degenerativas humanas.

Los pétalos de la flor amarilla del mastuerzo (Tropaeolum majus L.) son una fuente importante en carotenoides, encontrándose la presencia de $\beta$-caroteno, $\Upsilon$-caroteno, $\varepsilon$-caroteno y licopeno $^{1}$; con un potencial como fuente de compuestos antioxidantes. Sin embargo, al ser sometidas a calentamiento, pérdida de integridad celular, extracción, cambios de $\mathrm{pH}$, luz, temperatura; se tornan mucho más lábiles. 
A través de proceso de microencapsulación se puede estabilizar las propiedades nutracéuticas de los carotenoides extraídos de los pétalos del mastuerzo. La microencapsulación de compuestos bioactivos consiste en la extracción del compuesto activo, emulsificación y secado por atomización; este último, implica fenómenos de transferencia de calor y masa durante la formación de la microcápsula. La adición de agentes encapsulantes permiten el aumento del contenido de sólidos totales, reduciendo la humedad del producto, facilitando así el secado y reduciendo la pegajosidad. Las que deben evaluarse a fin de permitir el incremento de la estabilidad del producto protegiéndolo. El secado por spray-drying presenta la mayor retención de la actividad antioxidante ${ }^{2}$.

\section{PARTE EXPERIMENTAL}

\section{Materiales y reactivos}

Se trabajó con pétalos de mastuerzo color amarillo, recolectados de la estación experimental Mantaro de la Universidad Nacional del Centro del Perú. Ubicado en el distrito de Mantaro, provincia de Jauja, departamento de Junín.

Obtención del microencapsulado del extracto de carotenoides de los pétalos del mastuerzo (Tropaeolum majus L.) color amarillo

El extracto obtenido es exclusivamente de los pétalos de color amarillo de la flor de mastuerzo, una vez purificado, se mezcló con aceite de oliva, y se encapsuló con goma arábiga para luego ser llevado a secado por spray-drying, para tal fin se prosiguió el siguiente diagrama de flujo: 


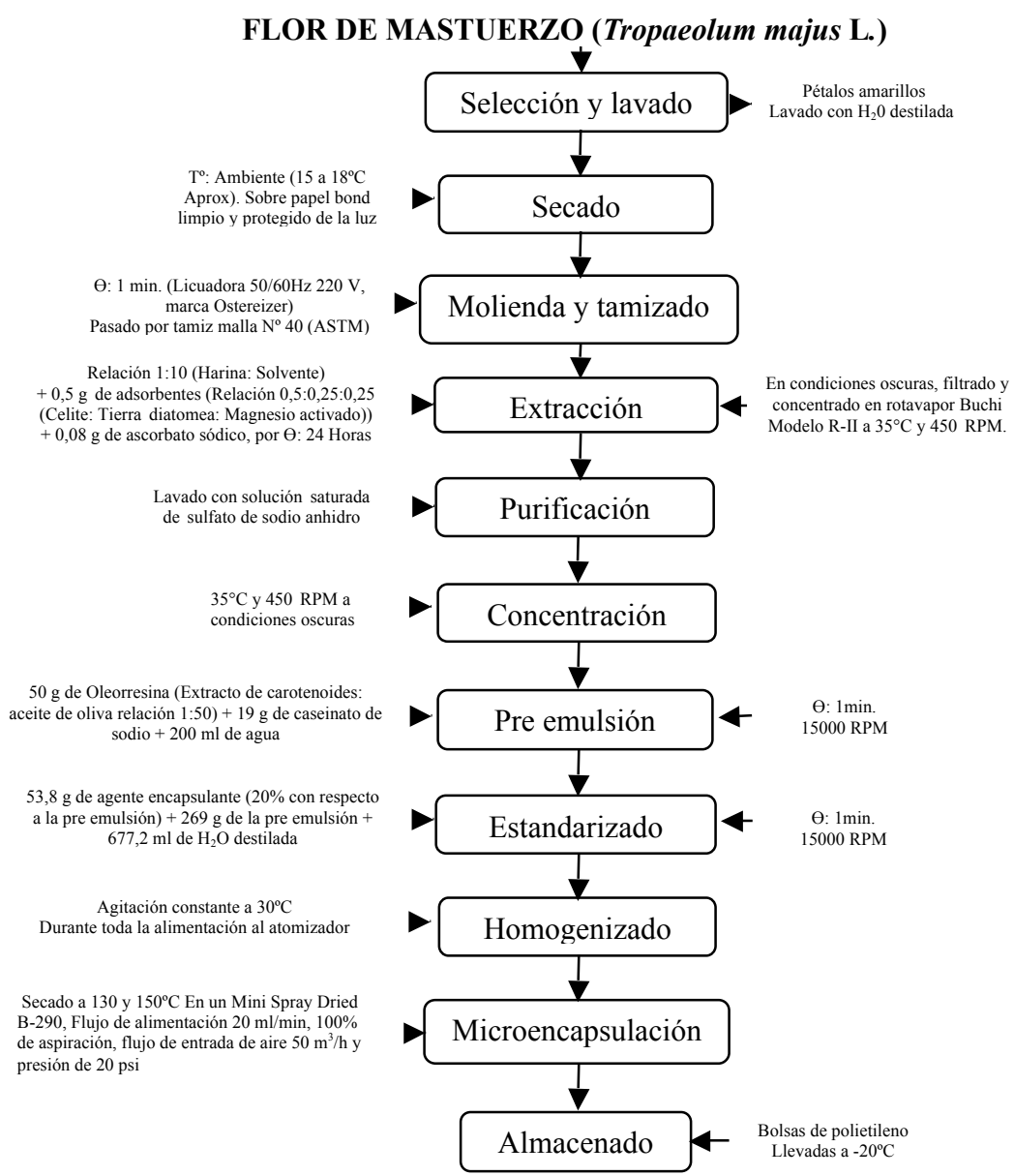

Figura 1. Diagrama de flujo de la microencapsulación por spray-drying del extracto de carotenoides los pétalos de mastuerzo (Tropaeolum majus L.) color amarrillo

\section{Cuantificación de carotenoides, actividad antioxidante, característica química y fisicoquímica}

En la cuantificación de los carotenoides totales, actividad antioxidante, característica química y fisicoquímica de la materia prima fresca, seca, extractos y las microcápsulas de carotenoides, se utilizó reactivos y protocolos debidamente validados.

\section{Determinación de carotenoides}

Carotenoides totales ${ }^{3}$

\section{Determinación de la actividad antioxidante}

Método $\mathrm{ABTS}^{4}$

Determinación de la característica química y fisicoquímica

Humedad $^{5}$, Higroscopicidad ${ }^{5}$, Solubilidad ${ }^{5}$, Densidad aparente ${ }^{5}$, Rendimiento. 
Recuperación de carotenoides: Su cálculo se basó en la cuantificación de carotenoides antes y después de encapsular hallándose el \% de recuperación mediante la siguiente fórmula:

$$
\% R=\frac{\text { Carotenoides totales después de encapsular }}{\text { Carotenoides totales antes de encapsular }} \times 100
$$

\section{Análisis estadístico}

Para la evaluación estadística se utilizó el DCA y el DCA con arreglo factorial de 2x3 cuyo análisis de varianza se trabajó con $p<0,05$ de significancia; al encontrar diferencia significativa $(p<0,05)$ se procedió a realizar la prueba de comparación de medias de Tukey. Los datos fueron procesados con la ayuda de los programas estadísticos Minitab V_16 y la hoja de cálculo Microsoft Excel 2010.

\section{RESULTADOS Y DISCUSIÓN}

\section{Cuantificación de carotenoides y actividad antioxidante en los pétalos de mastuerzo (Tropaeolum majus L.) color amarillo}

La cuantificación de los carotenoides totales y actividad antioxidante se efectuaron sobre los pétalos de mastuerzo (Tropaeolum majus L.) color amarillo en estado fresco con una humedad de $92,06 \%$ y estado seco con una humedad $11,23 \%$.

Tabla 1. Contenido de carotenoides totales y actividad antioxidante de los pétalos amarillos de mastuerzo (Tropaeolum majus L.).

\begin{tabular}{ccc}
\hline \hline Condición & $\begin{array}{c}\text { Carotenos totales } \\
(\mathrm{mg} \beta \text {-caroteno } / 100 \mathrm{~g} \text { en base Seca })\end{array}$ & $\begin{array}{c}\text { Actividad antioxidante } \\
(\mu \mathrm{mol}-\mathrm{TE} / \mathrm{g} \text { muestra en base seca })\end{array}$ \\
\hline \hline Fresco & $358,01 \pm 10,82^{\mathrm{a}}$ & $1922,34 \pm 23,05^{\mathrm{a}}$ \\
\multirow{2}{*}{ Seco } & $254,83 \pm 3,04^{\mathrm{b}}$ & $739,81 \pm 18,41^{\mathrm{b}}$ \\
\hline \hline
\end{tabular}

Valores con diferentes letras (a y b) dentro de cada columna denotan significancia en la prueba de Tukey $(p<0,05)$, valores promedio de 4 repeticiones \pm desviación estándar.

En la tabla 1 se observa que existe diferencia significativa $(p<0,05)$ entre las muestras, encontrándose un mayor contenido de carotenoides totales y actividad antioxidante en los pétalos frescos respecto a los deshidratados; Rodríguez-Amaya ${ }^{6}$ señala que en el lento secado de cuatro vegetales de hoja verde se dio una retención más baja de carotenoides, debido a la activación de las enzimas y esta aumenta al pulverizarla ya que se pierde la integridad causando la degradación e isomerización de los pigmentos amarillos que contienen $\beta$-carotenos, estos factores sumados al tiempo de almacenamiento son los principales responsables de su pérdida; con respecto a la actividad antioxidante del mastuerzo Garzón y Wrolstad ${ }^{7}$ reporta $458 \mu \mathrm{mol}-\mathrm{TE} / \mathrm{g}$ para flores naranjas; Arellano et al. ${ }^{8}, 587,01 \mu \mathrm{mol}-\mathrm{TE} / \mathrm{g}$ para flores amarillas; estos valores son menores a los hallados en la investigación; la variabilidad del contenido de carotenoides está influenciado por el clima, variedad, parte de la planta, estado de madurez, clima, cosecha, manejo post cosecha, procesamiento y almacenamiento ${ }^{6}$. 


\section{Cuantificación de carotenoides en los diferentes extractos de pétalos de mastuerzo (Tropaeolum majus L.) color amarillo}

La caracterización de los carotenoides totales en los diferentes extractos de pétalos de mastuerzo obtenido de los diferentes tiempos de extracción, se muestran en la tabla 2.

Tabla 2. Contenido de carotenoides totales ( $\mathrm{mg} \beta$-caroteno $/ 100 \mathrm{~g}$ muestra en base seca) de los diferentes extractos de los pétalos amarillos de mastuerzo (Tropaeolum majus $\mathrm{L}$ ).

\begin{tabular}{|c|c|c|}
\hline Extracto & Tiempos de extracción (Horas) & Carotenos totales \\
\hline \multirow{3}{*}{ Hexano } & 12 & $246,08 \pm 1,43^{f}$ \\
\hline & 24 & $256,87 \pm 1,06^{\mathrm{e}}$ \\
\hline & 48 & $329,14 \pm 1,02^{\mathrm{b}}$ \\
\hline \multirow{3}{*}{ Cloroformo } & 12 & $241,94 \pm 0,98^{\mathrm{g}}$ \\
\hline & 24 & $358,05 \pm 0,81^{\mathrm{a}}$ \\
\hline & 48 & $301,32 \pm 0,59^{c}$ \\
\hline \multirow{3}{*}{ Éter de petróleo } & 12 & $244,92 \pm 0,72^{\mathrm{fg}}$ \\
\hline & 24 & $258,4 \pm 1,46^{\mathrm{e}}$ \\
\hline & 48 & $297,97 \pm 1,14^{d}$ \\
\hline
\end{tabular}

Valores con diferentes letras (a y g) dentro de cada columna denotan significancia en la prueba de Tukey $(p<0,05)$, valores promedio de 4 repeticiones \pm desviación estándar.

En la tabla 2 se reporta el contenido de carotenoides totales por efecto de la interacción del solvente y tiempos de extracción las que muestran diferencias significativas $(p<0,05)$; encontrándose que el mejor tratamiento dio como resultado 358,05 $\pm 0,81 \mathrm{mg} \beta$-caroteno /100 g muestra, usando cloroformo durante un tiempo de lixiviación sólido-liquido de 24 horas; en la misma línea de investigación, Cornejo ${ }^{9}$ obtuvo la mejor extracción de carotenoides de la flor de Senna multiglandulosa con cloroformo comparado con éter etílico, isopropanol y éter de petróleo; Esta variabilidad en la composición de las mezclas de extracción está condicionada a la naturaleza y complejidad química de la fuente de pigmentos.

\section{Cuantificación de carotenoides en el extracto purificado de pétalos de mastuerzo (Tropaeolum majus L.) color amarillo}

La caracterización de los carotenoides totales del extracto purificado por saponificación en frío, saponificación en caliente y lavado se muestran en la tabla 3.

Tabla 3. Contenido de carotenoides totales ( $\mathrm{mg} \beta$-caroteno $/ 100 \mathrm{~g}$ muestra en base seca) de los extractos purificado de pétalos amarillos de mastuerzo (Tropaeolum majus L.).

\begin{tabular}{ccc}
\hline \hline Saponificación en caliente & Saponificación en frío & $\begin{array}{c}\text { Lavado con solución saturada de } \\
\text { sulfato de sodio anhidro }\end{array}$ \\
\hline \hline $230,53 \pm 1,81^{\mathrm{c}}$ & $236,47 \pm 2,93^{\mathrm{b}}$ & $319,33 \pm 3,25^{\mathrm{a}}$
\end{tabular}

Valores con diferentes letras (a y c) dentro de cada columna denotan significancia en la prueba de Tukey $(p<0,05)$, valores promedio de 4 repeticiones \pm desviación estándar. 
En la tabla 3 se reporta los resultados de los carotenoides totales de los extractos purificados en las que se aprecia que existe diferencia estadística $(p<0,05)$ en los distintos métodos, encontrándose que el mayor contenido de carotenoides se da en el lavado con solución saturada de sulfato de sodio anhidro teniendo entre un $25,95 \%$ a $27,80 \%$ de carotenoides totales adicionales con respecto a la saponificación en caliente y en frío; al respecto, Arellano ${ }^{10}$ señala que los carotenoides no son estables a pH extremos; ya que los álcalis pueden provocar isomerizaciones cis/trans de ciertos dobles enlaces, reagrupamientos y desesterificaciones al momento de saponificar, por lo que se recomienda no saponificar el extracto de pigmentos; el isomerismo de los pigmentos de los carotenoides empieza tan pronto como son solubilizados y no puede ser detenido por el almacenamiento en frío. Jaramillo ${ }^{11}$ afirma que el proceso de saponificación daña severamente a los pigmentos reflejándose en una fuerte disminución en la concentración de carotenoides totales; por otro lado, Gsakí y Martínez ${ }^{12}$ señalan que los procesos de lavados son muy frecuentes en las atapas de aislamiento y se llevan a cabo para eliminar restos orgánicos presentes en un mezcla orgánica de reacción. Rodríguez-Amaya ${ }^{6}$ comparó tres métodos de saponificación, con KOH en metanol al $10 \%$ y observó una pérdida de 0 a $3 \%$ a temperatura ambiente, mientras que con $\mathrm{KOH}$ en metanol al $60 \%$ se obtuvo mayores pérdidas y con el método de saponificación en caliente obtuvo pérdidas de hasta el $100 \%$, por lo que en la investigación se eligió el método del lavado para purificar el extracto de carotenoides.

\section{Cuantificación del \% de recuperación de carotenoides y actividad antioxidante de las microcápsulas del extracto de carotenoides de los pétalos de mastuerzo (Tropaeolum majus L.) color amarillo}

La cuantificación del \% de recuperación de carotenoides totales y la actividad antioxidante de las microcápsulas de carotenoides, obtenido del extracto purificado de carotenoides de los pétalos amarillos del mastuerzo, se muestran en la tabla 4.

Tabla 4. \% de recuperación de carotenoides y actividad antioxidante de las microcápsulas del extracto de carotenoides de los pétalos amarillos de mastuerzo (Tropaeolum majus L.).

\begin{tabular}{cccc}
\hline \hline $\begin{array}{c}\text { Agente } \\
\text { encapsulante }\end{array}$ & $\begin{array}{c}\text { Temperatura } \\
\text { secado }\end{array}$ & $\begin{array}{c}\text { \% Carotenos } \\
\text { totales recuperados }\end{array}$ & $\begin{array}{c}\text { Actividad antioxidante }(\mu \mathrm{mol}- \\
\text { TE/g muestra en base seca })\end{array}$ \\
\hline \hline Maltodextrina & $130^{\circ} \mathrm{C}$ & $54,82 \pm 0,03^{\mathrm{bc}}$ & $436,43 \pm 10,14^{\mathrm{ns}}$ \\
$(20 \%)$ & $150^{\circ} \mathrm{C}$ & $49,71 \pm 0,08^{\mathrm{c}}$ & $404,94 \pm 28,43^{\mathrm{ns}}$ \\
& $170^{\circ} \mathrm{C}$ & $45,16 \pm 0,02^{\mathrm{c}}$ & $398,00 \pm 3,37^{\mathrm{ns}}$ \\
\hline \multirow{2}{*}{ Goma arábiga } & $130^{\circ} \mathrm{C}$ & $83,76 \pm 0,06^{\mathrm{a}}$ & $470,97 \pm 20,74^{\mathrm{ns}}$ \\
$(20 \%)$ & $150^{\circ} \mathrm{C}$ & $74,10 \pm 0,01^{\mathrm{a}}$ & $418,32 \pm 22,45^{\mathrm{ns}}$ \\
& $170^{\circ} \mathrm{C}$ & $68,96 \pm 0,02^{\mathrm{ab}}$ & $393,38 \pm 72,47^{\mathrm{ns}}$
\end{tabular}

$\overline{* \text {, ns: indican significancia a } p<0,05 \text { y no significancia. Valores con diferentes letras (a y c) dentro de }}$ cada columna denotan significancia en la prueba de Tukey $(p<0,05)$, valores promedio de 3 repeticiones \pm desviación estándar. 
En la tabla 4 se reporta el \% de carotenoides totales por efecto de la interacción del agente encapsulante y temperatura de secado, en las que se evidencia diferencia significativa $(p<0,05)$. La mayor estabilidad de carotenoides se obtiene con el agente encapsulante goma arábiga y temperaturas de secado de $130{ }^{\circ} \mathrm{C}$ y $150{ }^{\circ} \mathrm{C}$ dándonos mayores $\%$ de recuperación de carotenoides. En la misma línea de investigación, Arellano ${ }^{10}$ encapsuló luteína de las flores de tagete (Tagete erecta) teniendo entre un 39,2 \% a 42,6 \% de recuperación usando como agente encapsulante la inulina y como oleorresina el aceite de girasol, estos niveles bajos de recuperación lo atribuye a las altas temperaturas de secado $\left(140{ }^{\circ} \mathrm{C}-180^{\circ} \mathrm{C}\right)$ ya que ocasionó la oxidación e isomerización de la luteína; Pedroza ${ }^{13}$ señala que el tipo de material encapsulante tiene influencia en la estabilidad de la emulsión antes de secar, en el tamaño de partícula, en las propiedades de flujo, en las mecánicas y en la vida útil del material deshidratado; asimismo, Matsumara et al. ${ }^{14}$, revelan que entre la interacción existente entre la goma arábiga y los lípidos esta última previene su oxidación, la primera tiene la propiedad de adsorberse en la interfase aceite/agua formando una película viscoelástica, donde los lípidos contribuyen con la coherencia de la estructura a través de la formación de empalmes o uniones por medio de gotitas de aceite en los anclajes de las cadenas de la goma arábiga; al respecto Larroza y Zerlotti ${ }^{15}$ señalan que la goma arábiga podría considerarse una excelente elección como material de pared para la encapsulación de licopeno por secado por aspersión. A ello, se le atribuye el mayor \% de recuperación de carotenoides en comparación del agente encapsulante maltodextrina, ya que la eficiencia en esta depende del grado de hidrólisis de las maltodextrinas, ya que muy bajos equivalentes de dextrosa no tienen el efecto positivo ${ }^{13}$.

En la actividad antioxidante no se evidencia diferencia estadística $(p>0,05)$ en los diferentes tratamientos encontrándose valores que van entre 393,38 a 470,97 $\mu$ mol-TE/g muestra; al respecto, Sáenz et al. ${ }^{2}$, en la microencapsulación de extracto etanólico de nopal (Opuntia ficus-indica) con inulina, reportan 61,7 $\mu \mathrm{mol}-\mathrm{TE} / \mathrm{g}$ atribuyendo la mayor actividad antioxidante al agente encapsulante; en misma línea de investigación, Arrazola, Herazo y Alvis $^{16}$, en la interacción de la variables temperatura de secado y \% de maltodextrina en los microencapsulados de extracto de antocianinas de la cáscara de berenjena, no encontraron diferencia estadística sobre la capacidad antioxidante.

\section{Caracterización de las microcápsulas del extracto de carotenoides de los pétalos de mastuerzo (Tropaeolum majus L.) color amarillo.}

La caracterización del \% de humedad, \% de higroscopicidad, \% solubilidad, densidad aparente $\left(\mathrm{g} / \mathrm{cm}^{3}\right)$ y el \% rendimiento se muestran en la tabla 5. 
Tabla 5. Características de las microcápsulas del extracto de carotenoides de los pétalos amarillos de mastuerzo (Tropaeolum majus L.).

\begin{tabular}{ccccccc}
\hline $\begin{array}{c}\text { Agente } \\
\text { encapsulante }\end{array}$ & $\begin{array}{c}\text { Temperatura } \\
\text { de secado }\end{array}$ & $\begin{array}{c}\text { Humedad } \\
\%\end{array}$ & $\begin{array}{c}\text { Higroscopicidad } \\
\%\end{array}$ & $\begin{array}{c}\text { Solubilidad } \\
\%\end{array}$ & $\begin{array}{c}\text { Densidad } \\
\text { aparente } \\
\left(\mathrm{g} / \mathrm{cm}^{3}\right)\end{array}$ & $\begin{array}{c}\text { Rendimiento } \\
\%\end{array}$ \\
\hline \hline & $130^{\circ} \mathrm{C}$ & $2,428 \pm 0,00^{\mathrm{a}}$ & $7,501 \pm 0,009^{\mathrm{c}}$ & $98,24 \pm 0,01^{\mathrm{ns}}$ & $1,068 \pm 0,01^{\mathrm{a}}$ & $19,20 \pm 0,01^{\mathrm{a}}$ \\
$\begin{array}{c}\text { Maltodextrina } \\
(20 \%)\end{array}$ & $150^{\circ} \mathrm{C}$ & $1,934 \pm 0,00^{\mathrm{b}}$ & $8,303 \pm 0,005^{\mathrm{c}}$ & $99,96 \pm 0,01^{\mathrm{ns}}$ & $1,044 \pm 0,01^{\mathrm{ab}}$ & $15,27 \pm 0,02^{\mathrm{b}}$ \\
\hline \hline & $170^{\circ} \mathrm{C}$ & $1,650 \pm 0,01^{\mathrm{b}}$ & $8,622 \pm 0,004^{\mathrm{bc}}$ & $99,02 \pm 0,01^{\mathrm{ns}}$ & $1,014 \pm 0,01^{\mathrm{bc}}$ & $18,79 \pm 0,02^{\mathrm{b}}$ \\
\hline $\begin{array}{c}\text { Goma arábiga } \\
(20 \%)\end{array}$ & $130^{\circ} \mathrm{C}$ & $2,285 \pm 0,01^{\mathrm{a}}$ & $10,811 \pm 0,006^{\mathrm{abc}}$ & $99,06 \pm 0,01^{\mathrm{ns}}$ & $0,946 \pm 0,02^{\mathrm{cd}}$ & $36,27 \pm 0,01^{\mathrm{a}}$ \\
& $150^{\circ} \mathrm{C}$ & $1,883 \pm 0,01^{\mathrm{bc}}$ & $11,866 \pm 0,002^{\mathrm{ab}}$ & $98,85 \pm 0,01^{\mathrm{ns}}$ & $0,961 \pm 0,01^{\mathrm{d}}$ & $23,35 \pm 0,01^{\mathrm{bc}}$ \\
& $170^{\circ} \mathrm{C}$ & $1,692 \pm 0,00^{\mathrm{cd}}$ & $14,144 \pm 0,018^{\mathrm{a}}$ & $99,15 \pm 0,01^{\mathrm{ns}}$ & $0,926 \pm 0,01^{\mathrm{d}}$ & $28,45 \pm 0,03^{\mathrm{cd}}$ \\
\hline
\end{tabular}

*, ns: indican significancia a $p<0,05$ y no significancia. Valores con diferentes letras (a y d) dentro de cada columna denotan significancia en la prueba de Tukey $(p<0,05)$, valores promedio de 3 repeticiones \pm desviación estándar.

En la tabla 5 se evidencia que existe diferencia significativa $(p<0,05)$ en cuanto al contenido de humedad, higroscopicidad, densidad aparente y rendimiento en los diferentes tratamientos por efecto de la interacción del agente encapsulante y la temperatura de secado. El contenido de humedad para el microencapsulado de carotenoides fue menor en los polvos obtenidos a altas temperaturas de secado; al respecto, Jittanit et al. ${ }^{17}$ señalan que a temperaturas más altas de entrada de aire, existe un gradiente de temperatura mayor entre la alimentación atomizada y el aire de secado, lo que resulta en una mayor fuerza motriz para la evaporación del agua, produciendo polvos con menor contenido de humedad; los valores de humedad obtenidos son cercanos a los reportados por Fernandes et al. ${ }^{18}$ en el secado de aceite esencial de romero que va de 0,26 a $3,16 \%$.

Con respecto a la higroscopicidad de las microcápsulas esta se incrementa a medida que se eleva la temperatura de secado, debido al menor contenido de humedad; Al respecto Tonon et al. ${ }^{19}$ señalan que hay una relación inversa entre la higroscopicidad y el contenido de humedad de los polvos y que está relacionado con el gradiente de concentración de agua entre el producto y el aire circundante; también se evidencia que la higroscopicidad varía con respecto al agente encapsulante, teniendo mayor higroscopicidad con el uso de goma arábiga; por otro lado, Caparino et al. ${ }^{20}$ mencionan que la maltodextrina permite obtener baja higroscopicidad en polvos, ello se corrobora en la investigación.

Se tienen una mayor densidad aparente de los polvos con la goma arábiga en comparación de la maltodextrina; al respecto, Jittanit et al. ${ }^{17}$ manifiestan que la temperatura de secado alta trae una mayor tasa de evaporación de la humedad de la alimentación dando como resultado una mayor porosidad y menor densidad aparente del microencapsulado; el agente encapsulante es un material de formación de cubierta y al ser utilizado induce a la acumulación y atrapamiento del aire dentro de la partícula haciendo que se vuelva menos densa y porosa ${ }^{20}$. 
Con respecto al rendimiento, se observa que el mayor rendimiento se obtuvo con la goma arábiga a $130^{\circ} \mathrm{C}$, se evidencia pérdidas a la hora de obtener el polvo entre 63,73 a $84,73 \%$; Salazar-González et al. ${ }^{21}$ atribuyen estas pérdidas al polvo que queda adherido en las paredes del secador, los mismos autores reportan rendimientos entre 71,3 a 74,9 \% en la producción de polvos del extracto de la flor de Jamaica usando goma de mezquite.

En cuanto a la solubilidad de los atomizados no se evidencia diferencia $(p>0,05)$ esta se incrementa a medida que la temperatura de entrada de aire es alta. La temperatura de secado tiene un efecto positivo sobre la solubilidad ya que altas temperaturas de secado dan como resultado una mayor porosidad de los polvos ${ }^{17}$. La mayor porosidad condujo a la superficie más específica del microencapsulado, dando como resultado la mayor superficie de contacto entre el polvo y el agua; Cano-Chauca et al. ${ }^{22}$ señalan que los portadores de secado maltodextrina y goma arábiga se disuelven fácilmente en agua, por ello mejoran la capacidad de disolución de las microcápsulas de carotenoides; la solubilidad del polvo secado está influenciada principalmente por el tipo y la concentración de los portadores de secado.

\section{CONCLUSIONES}

- El mejor método de extracción de carotenoides de pétalos amarillos de mastuerzo fue usando el cloroformo mediante lixiviación por un tiempo de 24 horas.

- La saponificación en frío y caliente del extracto de carotenoides de pétalos amarillos de mastuerzo (Tropaeolum majus L.) sobre el contenido de carotenoides totales, no es recomendable, por la alta degradación que implica este proceso.

- La microencapsulación de los extractos de carotenoides de los pétalos amarillos de mastuerzo con agente encapsulante goma arábiga y la temperatura de secado de $1300^{\circ} \mathrm{C}$ y $150^{\circ} \mathrm{C}$ permiten un mayor $\%$ de recuperación de carotenoides ( $74,10 \%$ y $\left.83,76 \%\right)$; estas presentan un alto potencial antioxidante $(418,32$ y $470,97 \mu \mathrm{mol}-\mathrm{TE} / \mathrm{g}$ muestra).

- La característica química y fisicoquímica de las microcápsulas de carotenoides permite establecer su uso como aditivo alimentario para su incorporación en alimentos funcionales.

\section{AGRADECIMIENTO}

Los autores expresamos nuestro agradecimiento al Instituto de Investigación de la UNCP, a la Facultad de Industrias Alimentarias y a los Fondos del Canon Minero administrados por la Universidad Nacional del Centro del Perú. 


\section{BIBLIOGRAFÍA}

1. Camavilca M, Leyva M. Identificación de antocianinas y carotenoides en flores de mastuerzo (Tropaeolum majus) por cromatografía en capa fina. [tesis para optar el titulo de Ingeniero en Industrias Alimentarias]. Huancayo: Universidad Nacional del Centro del Peru; 2015.

2. Saénz S, Tapia S, Chávez J, Robert P. Microencapsulation by spray drying of bioactive compounds from cactus pear (Opuntia ficus indica). Food Chem. 2009; 114(2): 616622.

3. Wrolstad R, Acree T, Decker E, Penner M, Reid D, Schwartz S, et al. Handbook of Analytical Chemistry: Pigments, Colorants, Flavor, Texture and Bioactive Food Components. Hoboken: Wiley Interscience, John Wiley \& Sons Inc. Publications; 2005.

4. Arnao M. Some methodological problems in the determination of antioxidant activity using chromogen radicals: A practical case. Trends Food Sci Technol. 2000; 11(11): 419-421.

5. Cai Y, Corke H. Production and properties of spray-dried Amaranthus betacyanin pigments. J Food Sci. 2000; 65(7): 1248-1252.

6. Rodriguez-Amaya D. Carotenoides y preparación de alimentos: retención de los carotenoides, provitamina A en alimentos preparados, procesados y almacenados. Primera edición. Campiñas: John Snow, Inc./OMNI; 1999.

7. Garzón G, Wrolstad R. Major anthocyanins and antioxidant activity of nasturtium flowers (Tropaeolum majus). Food Chem. 2009; 114(1): 44-49.

8. Arellano K, Herrera J, Quispe M, Espinoza C, Véliz N, Orihuela W. Evaluación de los compuestos fenólicos y capacidad antioxidante de tres colores de pétalos de mastuerzo (Tropaeolum majus L.). Rev Soc Quím Perú. 2015; 81(14): 319-328.

9. Cornejo D. Extracción, identificación, cuantificación y determinación de la actividad antioxidante de los carotenoides presentes en las flores de Senna Multiglandulosa a través de cuatro métodos de extracción. [tesis para optar el grado de Ingeniero en Biotecnologia]. Sangolqui: Escuela Politecnica del Ejército; 2011.

10. Arellano C. Extracción de luteína a partir de flores de tagete (Tagete erecta) y estabilización por microencapsulación. [tesis para optar el grado de Ingeniero en alimentos]. Santiago de Chile: Universidad de Chile; 2011.

11. Jaramillo E. Efecto del tratamiento térmico, enzimático y térmico-enzimático sobre los pigmentos y micro estructura de la flor de calabaza. Ciudad de México: Instituto Politécnico Nacional Escuela Nacional de Ciencias Biológicas; 2010.

12. Gsaky A, Martínez A. Técnicas experimentales en síntesis orgánica. Segunda edición. Madrid: Editorial Síntesis; 2012.

13. Pedroza R. Alimentos microencapsulados: Particularidades de los procesos para la microencapsulación de alimentos para larvas de especies acuícolas. Avances en Nutrición Acuícola VI. Memorias del VI Simposium Internacional de Nutrición Acuícola. CancúnMéxico; 2002.

14. Matsumara Y, Satake C, Egami M, Mori T. Interaction of gum arabic, maltodextrin and pullulan with lipids in emulsions. Biosci Biotechnol Biochem. 2000; 64(9): 1827-1835.

15. Larroza I, Zerlotti A. Encapsulation of Lycopene Using Spray Drying and Molecular Inclusion Processes. Braz Arch Biol Technol. 2007; 50(5): 893-900. 
16. Arrazola G, Herazo I, Alvis A. Microencapsulación de antocianinas de berenjena (Solanum melongena L.) mediante secado por aspersión y evaluación de la estabilidad de su color y capacidad antioxidante. Información tecnológica. 2014; 25(3): 31-42.

17. Jittanit W, Chantara-In M, Deying T, Ratanavong W. Production of tamarind powder by drum dryer using maltodextrin and Arabic gum as adjuncts. Songklanakarin J Sci Technol. 2011; 33 (1): 33-41.

18. Fernandes R, Borges S, Botrel D. Influencia de las condiciones de operación de secado por pulverización sobre propiedades de aceite esencial de romero microencapsuladas. Revista Ciencia tecnología de alimentos. 2013; 33(1): 171-178.

19. Tonon R, Brabet $\mathrm{C}$, Hubinger M. Influence of process conditions on the physicochemical properties of acai (Euterpe oleraceae Mart.) powder produced by spray drying. J Food Eng. 2008; 88 (3): 411-418.

20. Caparino O, Tang J, Nindo C, Sablani S, Powers J, Fellman J. Effect of drying methods on the physical properties and microstructures of mango (Philippine Carabao var.) powder. J Food Eng. 2012; 111(1): 135-148.

21. Salazar-GonzálezC, Vergara-Balderas F, Guerrero J.Evaluación de agentes encapsulantes de un extracto de Jamaica microencapsulado. Temas selectos de Ingeniería de Alimentos. 2009; 2(3): 14-25.

22. Cano-Chauca M, Stringheta L, Sardagna L, Cal-Vidal J. Mango juice dehydration spray drying using different carriers and functional characterization. Proceedings of the 14th International Drying Symposium, São Paulo, Brazil, 2004; 2005-2012. 